\title{
Confronto fra diversi modelli di calcolo per la valutazione del rischio sanitario-ambientale di un sito contaminato da idrocarburi
}

\author{
Noemi Cennamo ${ }^{1}$ \\ ${ }^{1}$ University of Salerno
}

\begin{abstract}
Il rischio è definito come la probabilità di insorgenza di effetti indesiderati per persone/cose, causati da particolari eventi, e può essere valutato come il prodotto tra la pericolosità (probabilità di accadimento dell'evento) e il danno atteso. Tra le differenti tipologie di rischio, nel presente lavoro si fa riferimento a quello ambientale. Il D.Lgs. 152/06, guarda al rischio sanitario-ambientale, derivante dall'impatto dei contaminanti presenti nelle matrici ambientali sulla salute umana. I siti contaminati costituiscono una problematica attuale e dunque si necessita di un'adeguata valutazione, al fine di individuare e quantificare i rischi associati ad un evento di contaminazione. L'obiettivo è effettuare un confronto tra software facendo riferimento ad un caso studio di un sito contaminato da idrocarburi in Spagna.
\end{abstract}

\section{La valutazione del rischio sanitario-ambientale nella legislazione}

\section{italiana e spagnola}

La bonifica dei siti contaminati rappresenta una priorità, non solo a livello nazionale, e di conseguenza altrettanto importante risulta l'esecuzione di un'adeguata analisi di rischio. In Italia, la normativa di riferimento è il D.Lgs. 152/06: non indica una specifica procedura di analisi di rischio 
da utilizzare, ma nell' Allegato 1 al Titolo V, Parte IV, sono riportati i “Criteri generali per l'analisi di rischio sanitario ambientale". Nel rispetto di tali criteri, l'analisi:

1. considera la componente probabilistica del rischio;

2. si fonda su criteri di conservatività;

3. richiede cura nella scelta dei parametri sito-specifici;

4. considera, ai fini dell'individuazione e dell'analisi dei possibili percorsi di esposizione, dei bersagli, nonché nella definizione degli obiettivi di bonifica, la destinazione d'uso (presente e futura) del sito.

L'analisi di rischio è lo strumento usato per stabilire se un sito è contaminato e quindi definire la necessità di attuare interventi di bonifica o di messa in sicurezza permanente. È inoltre sito-specifica ed applicata in modalità inversa, ovvero si procede con la stima della massima concentrazione $\left(\mathrm{C}_{\max }\right)$ alla sorgente, compatibile con le condizioni di accettabilità del rischio al bersaglio esposto, e successivamente al calcolo degli obiettivi di bonifica sito-specifici, imponendo come rischio incrementale (rischio causato dalla causa oggetto di studio) accettabile $10^{-6}$ per le sostanze cancerogene e 1 per quelle non cancerogene. Il D.Lgs. 152/06 prevede un'analisi che considera solo gli effetti della contaminazione sulla salute umana: la valutazione del rischio ecologico, ad oggi, non è prevista. I terreni contaminati in Spagna sono regolati dalla legge 22/2011, del 28 luglio e prevede la valutazione del rischio oltre che alla salute umana, anche quello di tipo ecologico ${ }^{1}$. Obiettivo del presente lavoro è il confronto dei risultati dell'analisi svolta, ottenuti implementando diversi software, eseguita su di un sito contaminato, prendendo come riferimento un caso studio. 


\section{Descrizione dei software impiegati e presentazione del caso stu-}

\section{dio}

La valutazione del rischio comprende la definizione della natura dei prodotti chimici esposti, l'entità, il tempo, i meccanismi di trasporto, le vie di esposizione e la sensibilità dei potenziali ricettori $^{2}$. In Europa sono stati sviluppati molti modelli e strumenti di valutazione del rischio per la salute umana, tra i quali CSOIL nei Paesi Bassi; Risk-net e ROME in Italia ${ }^{3},{ }^{4},{ }^{5},{ }^{6},{ }^{7}$ e a livello globale il kit di strumenti RBCA, sviluppato dall'American Society for Testing Materials (ASTM) negli Stati Uniti. Nel caso studio analizzato sono stati impiegati i kit di strumenti RBCA e CSOIL comunemente utilizzati in Spagna, e lo strumento Risk-net, basato sul modello ASTM-RBCA e adattato al framework italiano ${ }^{8}$. L'approccio del software RBCA è di tipo graduale (tiered), nel quale ogni gradino (tier) corrisponde ad un diverso livello di complessità e di conservatività. Il software Risk-net ricalca la procedura APAT-ISPRA, coerente con gli indirizzi della normativa italiana vigente ed infine il CSOIL consente di calcolare i rischi a cui sono esposti gli esseri umani se entrano in contatto con la contaminazione del suolo e anche la concentrazione massima di un contaminante nel terreno che non rappresenta un rischio per l'uomo.

\section{Procedura di analisi e valutazione del rischio del sito}

L'area di interesse è situata nei pressi di una stazione di rifornimento di benzina in un'area molto popolata. In un primo momento è stato effettuato il campionamento del suolo, successivamente sottoposto ad analisi di laboratorio con l'obiettivo di caratterizzarlo in termini di Total Petroleum Hydrocarbons (TPH). Dopo, per quei campioni che presentavano le concentrazioni più elevate, è stato valutato il rischio per la salute umana. Le ipotesi alla base della valutazione sono:

1. bambini come bersagli della contaminazione, essendo più sensibili ai contaminanti rispetto 
agli adulti;

2. destinazione d'uso del terreno residenziale (avendo considerato bambini come potenziali bersagli);

3. ingestione del suolo, contatto cutaneo, ingestione di acqua e inalazione di vapore come vie di esposizione alla contaminazione.

Sono stati prelevati otto campioni a profondità comprese tra 10 e 30 cm: sono stati estratti con una coclea a mano e conservati in apposite cassette catalogatrici, a basse temperature $\left(4^{\circ} \mathrm{C}\right)$, fino all' analisi.

Dopo la ricezione del campione, il terreno è stato trattato mediante liofilizzazione e setacciatura ${ }^{9}$. Come dimostrato in uno studio precedente ${ }^{10}$, concentrazioni inferiori a $200 \mathrm{mg} / \mathrm{kg}$ non presentano rischi inaccettabili, dunque per quei campioni che hanno mostrato concentrazioni di TPH superiori, è stato necessario effettuare il frazionamento ${ }^{10}$. Sulla base di studi precedenti ${ }^{11}$, sono state selezionate quattro frazioni di TPH alifatiche (EC 9-12, EC 12-16, EC 16-21 e EC 21-40) e quattro aromatiche (EC 10-12, CE 12-16, CE 16-21 e CE 21-35). Ogni campione è stato analizzato tre volte per poi considerare come valore finale la media dei tre risultati. Il contenuto di TPH e le sue frazioni sono stati quantificati con un gascromatografo GC2010 Shimadzu dotato di un rivelatore a ionizzazione di fiamma (GC - FID).

\section{Risultati dell'analisi}

Due campioni hanno mostrato le concentrazioni più elevate, 20 volte superiori rispetto al limite, per cui di questi ne è stata effettuata la valutazione del rischio e il frazionamento di TPH. Sono state rilevate tre diverse frazioni: EC21-40 alifatico, EC16-21 aromatico e EC21-35 aromatico. I software hanno permesso di valutare un Hazard Quotient (HQ) per singole frazioni non cancerogene, che sono riportate nella figura seguente. 
Per un campioni gli strumenti RBCA e Risk-net prevedono rischi inaccettabili per le due frazioni aromatiche, mentre la frazione alifatica presenta rischi inferiori a 1,0 dunque accettabili. Le stesse considerazioni possono essere estese ad un altro campione. Considerando invece i risultati ottenuti per le diverse frazioni e vie di esposizione, riportate nella figura successiva, si può affermare che:

- la frazione alifatica del campione più critico non presenta rischi inaccettabili per nessuno strumento e nessuna via di esposizione;

- la frazione aromatica di EC 16-21 presenta il rischio più elevato attraverso l'ingestione di acqua con gli strumenti RBCA e Risk-net, mentre lo CSOIL stima un valore molto inferiore;

- per la frazione aromatica EC21-35, gli strumenti RBCA e Risk-net considerando come via di esposizione più gravosa l'ingestione di acqua presentando anche risultati simili, mentre per lo strumento CSOIL, la via di esposizione più importante è l'ingestione del suolo. Le stesse riflessioni valgono per i campioni per il quali si sono registrati valori leggermente più bassi perché le concentrazioni iniziali delle diverse frazioni di TPH erano inferiori rispetto a quelle del campione con concentrazione maggiore.

Per la via di esposizione di ingestione del suolo, gli strumenti RBCA e Risk-net forniscono un valore di TPH analogo perché applicano la stessa equazione, e questo risulta essere circa il doppio del risultato dello CSOIL: il parametro che produce la differenza è il tasso di ingestione del suolo il cui valore USA è il doppio del valore olandese. Per quanto riguarda la via di esposizione di ingestione di acqua contaminata si osservano valori più alti e simili considerando i modelli RBCA e Risk-net, differentemente dal modello CSOIL che ha riportato un valore molto più basso: la differenza è motivata dal fatto che i modelli RBCA e Risk-net considerano che i contaminanti del suolo possano lisciviare e influenzare le acque sotterranee successivamente consumate dall'uomo, mentre il modello CSOIL ritiene che le acque contaminate non vengano ingerite direttamente, ma che una tubazione possa trovarsi a contatto con acque sotterranee contaminate e che quindi le sostanze 
possano penetrare attraverso la tubazione, ma al contempo che le acque vengano trattate prima di essere distribuite. Poiché la pressione di vapore delle frazioni rilevate sono risultate basse tali da evitare la dispersione nell' aria ${ }^{12}$, questa non è stata considerata come possibile via di migrazione del contaminante.

\section{Conclusioni}

Il presente lavoro ha riguardato il confronto dei risultati ottenuti, impiegando diversi software, per la valutazione del rischio sanitario-ambientale. Nello studio è stata descritta inizialmente la fase di campionamento del suolo e delle analisi condotte in laboratorio dei campioni. Due dei campioni estratti hanno registrato una concentrazione superiore al valore limite e dunque sono stati sottoposti a frazionamento e alla valutazione del rischio, con i software RBCA, CSOIL e Risk-net. Dai risultati riportati nel caso studio è emerso che:

1. gli strumenti RBCA e Risk-net, seguendo un approccio simile, hanno riportato risultati confrontabili, indicando come via di esposizione principale, l'ingestione di acqua potabile;

2. secondo il modello CSOIL, la principale via di esposizione ai contaminanti è costituita dall'ingestione di terreno.

3. La differenza tra i modelli riguarda sostanzialmente l'approccio: RBCA e Risk-net sono utili per scenari in cui le acque sotterranee interessate possono essere ingerite direttamente, mentre CSOIL considera che i contaminanti possano penetrare nella tubazione che trasporta l'acqua, contaminandola, ma che poi questa venga trattata prima di essere distribuita.

4. Il modello CSOIL rappresenta le condizioni effettive di valutazione del rischio del sito, registrando valori del rischio accettabili

Nonostante le conclusioni del caso studio riportino che il software CSOIL sia il modello più adatto, in virtù dei risultati prodotti (accettabile a differenza degli altri due modelli) e per le ipotesi su cui 
si fonda, è possibile affermare che esso potrebbe risultare troppo poco conservativo, e per questo fornire risultati non particolarmente affidabili.

\section{References}

1.D’Aprile, L. Bonifiche e normativa: stato dell'arte. Ecoscienza 3, (2010).

2.USEPA. Human Health Risk Assessment Strategic Research Action Plan 2012-2016 — US EPA. in (EPA Research, 2012).

3.APAT. Methodological Criteria for Risk Assessment Application to Contaminated Sites (Rev.2). in Environmental Protection and Technical Service Agency, Rome, Italy (http://www.isprambiente.gov.it/files/temi/siti-contaminati02marzo08.pdf, 2008).

4.ASTM. Guide for Risk-Based Corrective Action Applied at Petroleum Release Sites. in (ASTM International, 2004). doi:10.1520/e1739-95e01.

5.Brand, E., Otte, P. F. \& Lijzen, J. P. A. CSOIL 2000 an Exposure Model for Human Risk Assessment of Soil Contamination. A Model Description. National Institute for Public Health and the Environment (RIVM), Bilthoven, The Netherlands (2007).

6.Carlon, C. Derivation methods of soil screening values in Europe. A review and evaluation of national procedures towards harmonisation - ESDAC - European Commission. (https://esdac.jrc.ec.europa.eu/content/derivation-methodssoil-screening-values-europe-review-and-evaluation-nationalprocedures, 2007).

7.Connor, J. A., Bowers, R. L., McHugh, T. E. \& Spexet, A. M. H. RBCA Software Tool Kit for 
Chemical Releases Version 2.6. (GSI Environmental, Inc., Houston, Texas, USA, 2009).

8.Pinedo, J., Ibáñez, R. \& Irabien, Á. A comparison of models for assessing human risks of petroleum hydrocarbons in polluted soils. Environmental Modelling \& Software 55, 61-69 (2014).

9.ISO. ISO 2005a. in (International Organization for Standardization, Geneva, Switzerland, 2005).

10.Pinedo, J., Ibáñez, R., Lijzen, J. P. A. \& Irabien, Á. Human Risk Assessment of Contaminated Soils by Oil Products: Total TPH Content Versus Fraction Approach. Human and Ecological Risk Assessment: An International Journal 1231-1248 (2013) doi:10.1080/10807039.2013.831264.

11.Pinedo, J., Ibañez, R., Primo, O. \& Irabien, A. Hydrocarbons Analysis for Risk Assessment in Polluted Soils — Semantic Scholar. Chemical Engineering Transactions 28, (2012).

12.ATSDR. Toxicological Profile for Total Petroleum Hydrocarbons (TPH). in (U.S. Department of Health and Human Services, Public Health Service, Atlanta, Georgia, USA, 1999). 
Figure Captions

Figure 1. Raccolta ed analisi di data set 
Figures

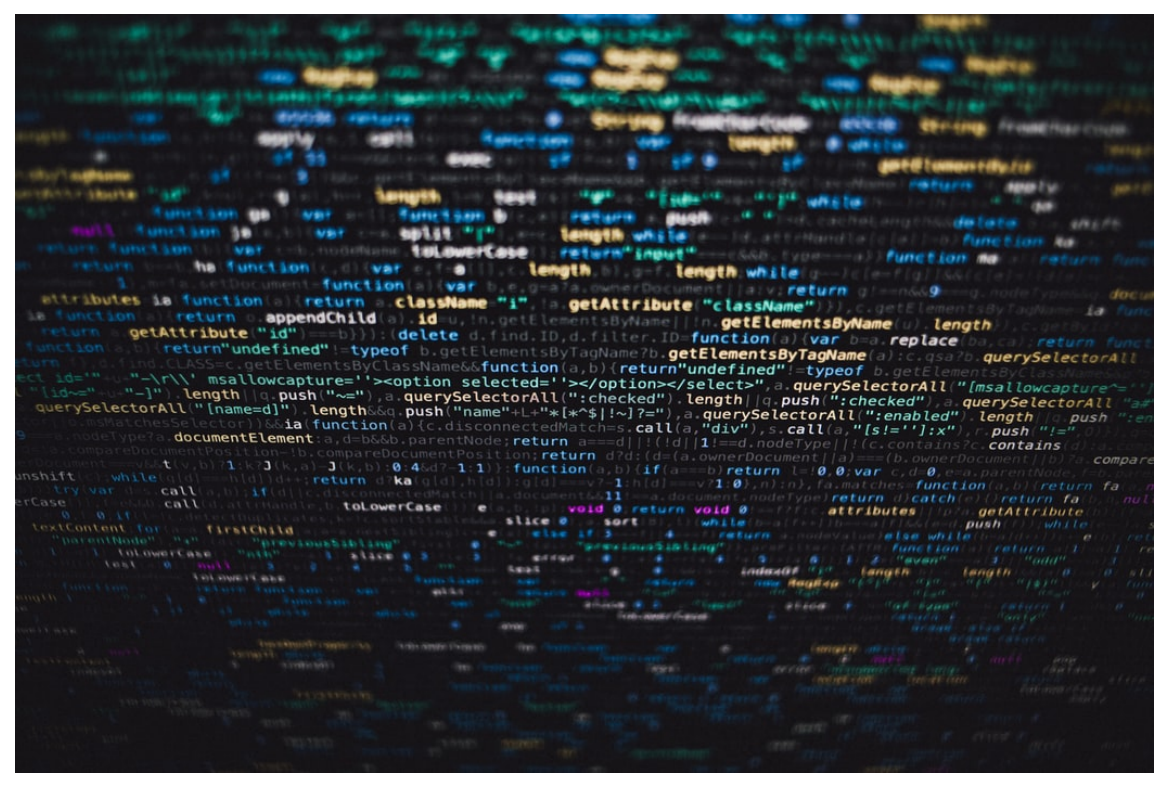

Figure 1: Raccolta ed analisi di data set 